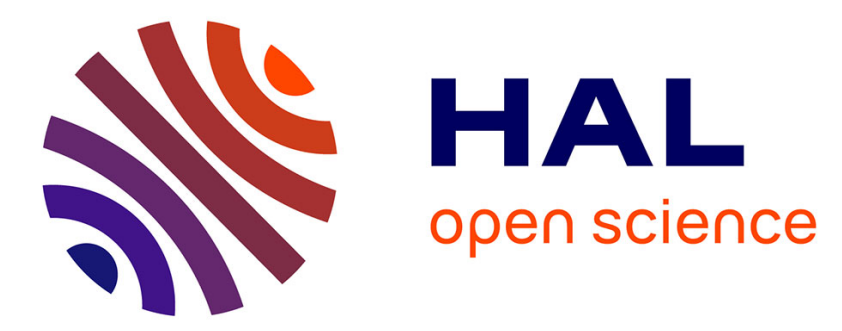

\title{
Limiting Power Transmission of Green Cellular Networks: Impact on Coverage and Capacity
} Jean-Marc Kélif, Marceau Coupechoux, Frédéric Marache

\section{To cite this version:}

Jean-Marc Kélif, Marceau Coupechoux, Frédéric Marache. Limiting Power Transmission of Green Cellular Networks: Impact on Coverage and Capacity. IEEE International Conference on Communications, ICC, May 2010, Cape Town, South Africa. pp.1-6. hal-01144498

\section{HAL Id: hal-01144498 https://hal-imt.archives-ouvertes.fr/hal-01144498}

Submitted on 21 Apr 2015

HAL is a multi-disciplinary open access archive for the deposit and dissemination of scientific research documents, whether they are published or not. The documents may come from teaching and research institutions in France or abroad, or from public or private research centers.
L'archive ouverte pluridisciplinaire HAL, est destinée au dépôt et à la diffusion de documents scientifiques de niveau recherche, publiés ou non, émanant des établissements d'enseignement et de recherche français ou étrangers, des laboratoires publics ou privés. 


\section{Limiting Power Transmission of Green Cellular Networks: Impact on Coverage and Capacity}

\author{
Jean-Marc Kelif \\ Orange Labs \\ Issy-Les-Moulineaux, France \\ jeanmarc.kelif@ orange-ftgroup.com
}

\author{
Marceau Coupechoux \\ Télécom ParisTech and CNRS LTCI \\ 46, rue Barrault, Paris, France \\ coupecho@enst.fr
}

\author{
Frédéric Marache \\ Orange Labs \\ Issy-Les-Moulineaux, France \\ frederic.marache@orange-ftgroup.com
}

\begin{abstract}
Reducing power transmission is of primary importance in future green cellular networks. First of all, the induced reduction of the interference encourages the deployment of opportunistic radios in the same spectrum. Then, it directly implies a reduction of the energy consumption. At last, electric field radiations reduction mitigates the potential risks on health. From a technical point of view, power control is however likely to degrade network performance. In this paper, we evaluate the impact of power reduction on the coverage and the capacity of cellular networks. We establish closed form formulas of outage probability by taking into account shadowing, thermal noise and base stations (BS) transmitting power impacts. We quantify the transmitting power needed for different kinds of environments (urban, rural) and frequencies and we show that the transmitting power can be optimized according to networks characteristics without decreasing the quality of service. We show at last that increasing the BS density results in a reduction of the global power density in the network.
\end{abstract}

\section{INTRODUCTION}

An important challenge of future wireless networks consists in decreasing the base station (BS) transmitting powers with a limited impact on the quality of service. This limitation allows indeed to decrease (1) the amount of interference in the considered spectrum; (2) the energy consumption with aim of preserving the environment and reducing the cost of the network; and (3) the electromagnetic pollution in order to mitigate potential risks on human health. For these reasons, power control appears to be the main feature of green cellular networks.

However, a BS transmitting power limitation may have a negative impact on the network performance and quality of service. Both interference and useful signal strength indeed decrease. In this paper, we propose an analytical approach to quantify at a click speed the impact of transmitting power on capacity and coverage.

The estimation of cellular networks capacity and coverage mainly depends on the characterization of interference. In this paper, we focus on the downlink, but the proposed framework can easily be extended to the uplink. An important parameter for estimating interference is the other-cell interference factor $f$ (OCIF). The precise knowledge of the OCIF allows the derivation of outage probabilities and Signal to Interference plus Noise Ratio (SINR) distributions, needed for coverage and capacity evaluations. In this paper, we define OCIF as the ratio of total other-cell received power to the total inner-cell received power.

Working on the uplink, [12] derived the distribution function of a ratio of path-losses, which is essential for the evaluation of external interference. For that, authors approximate the hexagonal cell with a disk of same area. On the downlink, [1] [2] aimed at computing an average OCIF over the cell by numerical integration in hexagonal networks. In [11], othercell interference is given as a function of the distance to the base station (BS) thanks to Monte-Carlo simulations. Chan and Hanly [10] precisely approximate the distribution of the other-cell interference. They however provide formulas that are difficult to handle in practice. Reference [7] computes the outage probability in presence of multiple log-normal interferers, but assumes that all interference signals have the same statistics and mobile location influence is not studied.

Coverage studies in cellular networks, especially when frequency reuse is one, often neglect the impact of noise. This is the case in [10], [11], [7]. In [3] also, outage probability calculation does not consider thermal noise. This is a reasonable assumption for typical output powers and in urban environments. It is however questionable at high frequencies, in rural environments or when BS output power is decreased.

In this paper, we give a new perspective on the influence of BS transmission power and thermal noise in different environments and for different frequencies. We derive closed form formulas for the outage probability with and without shadowing and we analyze the effect of decreasing BS power on coverage and capacity. We use the expressions of the OCIF without shadowing established in [8] and with shadowing established in [14] to calculate analytically the outage probability by using a Gaussian approximation.

In the next section, we first introduce the model and the notations. In section III] we establish outage probability formulas. In sections IV and D, we analyze the interference factor and the thermal noise contribution. In section VI, we propose an application to green networks and show that it is possible to decrease the transmitting power of a wireless network with a limited impact on the quality of service.

\section{INTERFERENCE MODEL AND NOTATIONS}

We consider a cellular radio system and we focus on the downlink. BS have omni-directional antennas, so that a BS 
covers a single cell. If a mobile $u$ is attached to a station $b$ (or serving $\mathrm{BS}$ ), we write $b=\psi(u)$.

The following power quantities are considered:

- $P_{b, u}$ is the transmitted power from station $b$ towards mobile $u$ (for user's traffic),

- $P_{b}=P_{c c h}+\Sigma_{u} P_{b, u}$ is the total power transmitted by station $b$,

- $P_{c c h}$ represents the amount of power used to support broadcast and common control channels,

- $g_{b, u}$ designates the path-gain between station $b$ and mobile $u$,

- $p_{b, u}$ is the power received at mobile $u$ from station $b$, we can write $p_{b, u}=P_{b} g_{b, u}$

- $S_{b, u}=P_{b, u} g_{b, u}$ is the useful power received at mobile $u$ from station $b$ (for traffic data); since we do not consider soft handover, we can write $S_{b, u}=S_{\psi(u), u}=S_{u}$.

We consider a reuse 1 radio system such as OFDMA or CDMA. The total amount of power experienced by a mobile station $u$ in a cellular system can be split up into several terms: useful signal $\left(S_{u}\right)$, interference and noise $\left(N_{t h}\right)$. It is common to split the system power into two terms: $I_{u}=I_{\text {int }, u}+I_{\text {ext }, u}$, where $I_{i n t, u}$ is the internal (or own-cell) received power and $I_{\text {ext }, u}$ is the external (or other-cell) interference. Notice that we made the choice of including the useful signal $S_{u}$ in $I_{i n t, u}$, and, as a consequence, it has to be distinguished from the commonly considered own-cell interference.

With the above notations, we define the interference factor in $u$, as the ratio of total power received from other BS to the total power received from the serving BS $\psi(u): f_{u}=$ $I_{e x t, u} / I_{i n t, u}$. The quantities $f_{u}, I_{e x t, u}$, and $I_{i n t, u}$ are location dependent and can thus be defined in any location $x$ as long as the serving BS is known.

In downlink, a coefficient $\alpha$, may be introduced to account for the lack of perfect orthogonality in the own cell. In a CDMA system $0<\alpha<1$, and in an OFDMA system $\alpha=0$.

In this paper, we use the signal to interference plus noise ratio (SINR) as the criteria of radio quality: $\gamma_{u}^{*}$ is the SINR target for the service requested by MS $u$ or required to obtain a minimum throughput in case of elastic traffic. This figure is a priori different from the SINR evaluated at mobile station $u$. However, we assume perfect power control, so $S I N R_{u}=\gamma_{u}^{*}$ for all users. With the introduced notations, the SINR experimented by $u$ can thus be derivated (see e.g. [4]):

$$
\gamma_{u}^{*}=\frac{S_{u}}{\alpha\left(I_{i n t, u}-S_{u}\right)+I_{e x t, u}+N_{t h}} .
$$

In case of OFDMA, this equation is valid per sub-carrier. We can now express $S_{u}$ as:

$$
S_{u}=\frac{\gamma_{u}^{*}}{1+\alpha \gamma_{u}^{*}} I_{i n t, u}\left(\alpha+I_{e x t, u} / I_{i n t, u}+N_{t h} / I_{i n t, u}\right) .
$$

Using relations $I_{i n t, u}=P_{b} g_{b, u}$ and $f_{u}=I_{e x t, u} / I_{i n t, u}$ we can write

$$
f_{u}=\frac{\sum_{j \neq b} P_{j} g_{j, u}}{P_{b} g_{b, u}}
$$

and the transmitted power for MS $u, P_{b, u}=S_{u} / g_{b, u}$,

$$
P_{b, u}=\frac{\gamma_{u}^{*}}{1+\alpha \gamma_{u}^{*}}\left(\alpha P_{b}+f_{u} P_{b}+N_{t h} / g_{b, u}\right) .
$$

From this relation, the output power of BS $b$ can be computed as follows:

$$
P_{b}=P_{c c h}+\sum_{u} P_{b, u}
$$

and so, according to $\mathrm{Eq} 4$

$$
P_{b}=\frac{P_{c c h}+\sum_{u} \frac{\gamma_{u}^{*}}{1+\alpha \gamma_{u}^{*}} \frac{N_{t h}}{g_{b, u}}}{1-\sum_{u} \frac{\gamma_{u}^{*}}{1+\alpha \gamma_{u}^{*}}\left(\alpha+f_{u}\right)} .
$$

Note that this equation is valid for multiple SINR targets and thus for multi-service networks since $\gamma_{u}^{*}$ is specific to user $u$. In the rest of this paper, we will however consider a single service $\left(\gamma_{u}^{*}=\gamma^{*} \forall u\right)$ and an homogeneous network $\left(P_{j}=\right.$ $\left.P_{b} \forall j\right)$.

In the following sections, we will consider two channel models. Without shadowing, $g_{b, u}=K r_{b, u}^{-\eta}$. With shadowing, $g_{b, u}=K r_{b, u}^{-\eta} A_{b}$, where $A_{b}=10^{-\xi_{b} / 10}$ is a lognormal random variable $(\mathrm{RV})$ with logarithm mean and standard deviation $0 \mathrm{~dB}$ and $\sigma(\mathrm{dB})$ respectively and $K$ is constant which depends on the frequency.

Noise power can be expressed as $N_{t h}=N_{0} W$, where $N_{0}$ is the noise power spectral density and $W$ is the system bandwidth.

Index $b$ will be sometimes dropped when no confusion is possible, so that $r_{u}=r_{b, u}$.

\section{OUTAGE PROBABILITIES}

For a given number of MS per cell, $n$, outage probability, $P_{\text {out }}^{(n)}$, is the proportion of configurations, for which the needed BS output power exceeds the maximum output power: $P_{b}>$ $P_{\max }$ :

$$
P_{\text {out }}^{(n)}=\operatorname{Pr}\left[\sum_{u=0}^{n-1} f_{u}+h_{u}>\frac{1-\varphi}{\beta}-n \alpha\right]
$$

where $\varphi=P_{c c h} / P_{\max }, \beta=\gamma^{*} /\left(1+\alpha \gamma^{*}\right)$ and $h_{u}=$ $\frac{N_{t h}}{P_{\max } g_{b, u}}$. The first term of the left hand side of the inequality represents the impact of the interferences due to the other base stations of the system. The second term represents the impact of the thermal noise and BS output power. We denote $T_{u}$ the following term:

$$
T_{u}=f_{u}+h_{u}
$$

In order to compute the outage probability, we rely on the Central Limit theorem and use the Gaussian approximation. We have thus to compute the mean and standard deviation of $T_{u}$. 


\section{A. Without shadowing}

Let first assume that there is no shadowing. Let $\mu_{T}$ and $\sigma_{T}$ be the mean and standard deviation of $T_{u}$. The outage probability can be approximated by:

$$
P_{\text {out }}^{(n)}=Q\left(\frac{\frac{1-\varphi}{\beta}-n \mu_{T}-n \alpha}{\sqrt{n} \sigma_{T}}\right)
$$

Knowing mobile location $r_{u}$, the interference factor is here a deterministic function. However, mobile locations are random. Hence, variable $T_{u}$ is random and depends on the locations of the $n$ mobiles in the cell. Without shadowing, we denote $f_{u}=f_{0}\left(r_{u}\right)$ the interference factor and $h_{u}=h_{0}\left(r_{u}\right)$ the thermal factor.

Without shadowing, we have thus:

$$
\begin{aligned}
\mu_{T} & =\mu_{f_{0}}+\mu_{h_{0}} \\
\sigma_{T}^{2} & =\sigma_{f_{0}}^{2}+\sigma_{h_{0}}^{2}+2 \mathbb{E}\left[f_{0} h_{0}\right]-2 \mu_{f_{0}} \mu_{h_{0}}
\end{aligned}
$$

where $\mu_{f_{0}}$ and $\sigma_{f_{0}}$ are respectively the spatial mean and standard deviation of $f_{0}, \mu_{h_{0}}$ and $\sigma_{h_{0}}$ the spatial mean and standard deviation of $h_{0}$ and the expectation is taken on the uniform distribution of mobiles over the cell area.

\section{B. Shadowing impact}

Let us now consider the shadowing impact. For each location $r_{u}, f_{u}$ is a random variable because of shadowing. Mobiles are still assumed to be randomly located in the cell. Hence, variable $T_{u}$ is random and depends both on shadowing and on mobile location. Using the Gaussian approximation, the outage probability is expressed as:

$$
P_{\text {out }}^{(n)}=Q\left(\frac{\frac{1-\varphi}{\beta}-n M_{T}-n \alpha}{\sqrt{n} S_{T}}\right)
$$

where $M_{T}$ and $S_{T}$ are respectively the spatial mean and standard deviation of the random variable $T_{u}$.

With shadowing, we have:

$$
\begin{aligned}
M_{T} & =M_{f}+M_{h}, \\
S_{T}^{2} & =S_{f}^{2}+S_{h}^{2}+2 \mathbb{E}\left[f_{u} h_{u}\right]-2 M_{f} M_{h}
\end{aligned}
$$

where $M_{f}, S_{f}, M_{h}$ and $S_{h}$ are the spatial mean and standard deviation respectively of random variables $f_{u}$ and $h_{u}$. The expectation is taken both on shadowing variations and mobile location. In the next section, we now characterize the interference factor more precisely.

\section{INTERFERENCE FACTOR ANALYSIS}

In this section, we compute the mean and standard deviations of $f_{u}$ without shadowing $\left(\mu_{f_{0}}\right.$ and $\left.\sigma_{f_{0}}\right)$ and with shadowing $\left(M_{f}\right.$ and $\left.S_{f}\right)$.

\section{A. Without shadowing}

1) Interference factor at distance $r_{u}$ : Forgetting in this section the shadowing impact, Eq. 3 can be written:

$$
f_{0}=\frac{\sum_{j \neq b} r_{j, u}^{-\eta}}{r_{b, u}^{-\eta}}
$$

For a mobile at the distance $r_{u}$ from its serving base station, the interference factor can be written as $f_{0}[8]$ [13]:

$$
f_{0}=\frac{2 \pi \rho_{B S} r^{\eta}}{\eta-2}\left(2 R_{c}-r\right)^{2-\eta}
$$

where $1 / \rho_{B S}=\pi R_{e}^{2}$ represents the cell area and $2 R_{c}$ the distance between two neighbour BS. We have $R_{e}=R_{c} \sqrt{2 \sqrt{3} / \pi}$.

2) Integration over the cell area: In order to compute $\mu_{f_{0}}$ and $\sigma_{f_{0}}$, we integrate $f_{0}$ on a disk of radius $R_{e}$. As MS are uniformly distributed over the disk of radius $R_{e}$, the PDF of $r$ is: $p_{r}(r)=\frac{2 r}{R_{e}^{2}}$.

$$
\begin{aligned}
\mu_{f_{0}}= & \frac{2 \pi \rho_{B S}}{\eta-2} \int_{0}^{R_{e}} r^{\eta}\left(2 R_{c}-r\right)^{2-\eta} \frac{2 r}{R_{e}^{2}} d r \\
= & \frac{2^{4-\eta} \pi \rho_{B S} R_{c}^{2}}{\eta^{2}-4}\left(\frac{R_{e}}{R_{c}}\right)^{\eta} \times \\
& { }_{2} F_{1}\left(\eta-2, \eta+2, \eta+3, R_{e} / 2 R_{c}\right),
\end{aligned}
$$

where ${ }_{2} F_{1}(a, b, c, z)$ is the hypergeometric function, whose integral form is given by:

$$
{ }_{2} F_{1}(a, b, c, z)=\frac{\Gamma(c)}{\Gamma(b) \Gamma(c-b)} \int_{0}^{1} \frac{t^{b-1}(1-t)^{c-b-1}}{(1-t z)^{a}} d t,
$$

and $\Gamma$ is the gamma function.

In the same way, the variance of $f_{0}$ is given by:

$$
\begin{aligned}
\sigma_{f_{0}}^{2}= & E\left[f_{0}^{2}\right]-\mu_{f_{0}}^{2} \\
E\left[f_{0}^{2}\right]= & \frac{2^{4-2 \eta}\left(2 \pi \rho_{B S} R_{c}^{2}\right)^{2}}{(\eta+1)(\eta-2)^{2}}\left(\frac{R_{e}}{R_{c}}\right)^{2 \eta} \times \\
& { }_{2} F_{1}\left(2 \eta-4,2 \eta+2,2 \eta+3, \frac{R_{e}}{2 R_{c}}\right) .
\end{aligned}
$$

\section{B. Shadowing impact}

1) Random variable at distance $r_{u}$ : Let us now assume the presence of shadowing. Recall that the interference factor is defined as $f_{u}=I_{e x t, u} / I_{i n t, u}$. On one hand, $I_{e x t, u}=$ $\sum_{j \neq b}^{B} P_{b} g_{j, u}$ is a sum of lognormal RV. Such a sum can be approximated by another lognormal distribution [3] using the Fenton-Wilkinson [5] method.

On the other hand, $I_{i n t, u}=P_{b} g_{b, u}$ is a lognormal RV. As a ratio of two lognormal $\mathrm{RV}$, the interference factor $f_{u}$ can thus be approximated by a lognormal RV with parameters $m_{f}$ and $s_{f} \cdot \ln \left(f_{u}\right)$ is thus a normal RV expressed as $N\left(m_{f}, a^{2} s_{f}^{2}\right)$, where $a=\ln (10) / 10$.

We introduced in [14]

$$
L\left(r_{u}, \eta\right)=\frac{f_{0}\left(r_{u}, 2 \eta\right)}{f_{0}\left(r_{u}, \eta\right)^{2}}
$$


and

$$
J\left(r_{u}, \sigma\right)=e^{a^{2} \sigma^{2} / 2}\left(L\left(r_{u}, \eta\right)\left(e^{a^{2} \sigma^{2}}-1\right)+1\right)^{-\frac{1}{2}},
$$

and showed that

$$
\begin{aligned}
m_{f} & =\ln \left(f_{0}\left(r_{u}, \eta\right) J\left(r_{u}, \sigma\right)\right), \\
a^{2} s_{f}^{2} & =2\left(a^{2} \sigma^{2}-\ln J(r, \sigma)\right),
\end{aligned}
$$

when received powers are uncorrelated. We also showed that

$$
\begin{aligned}
m_{f, c o r r} & =\ln \left(f_{0}\left(r_{u}, \eta\right) J\left(r_{u}, \sigma_{g}\right)\right), \\
a^{2} s_{f, c o r r}^{2} & =2\left(a^{2} \sigma_{g}^{2}-\ln J\left(r, \sigma_{g}\right)\right)+2 t a^{2} \sigma^{2},
\end{aligned}
$$

when received power are correlated with a correlation coefficient $t$ and a correlated variance $\sigma_{g}^{2}=\sigma^{2}(1-t)$.

2) Integration over the cell area: We now integrate the RV $f_{u}$ over the cell area assuming a uniform distribution of the mobiles. The mean $\mathbb{E}\left[f_{u}\right]$ of the interference factor can be written:

$$
\begin{aligned}
M_{f} & =\int_{0}^{R_{e}} \mathbb{E}\left[f_{u} \mid r\right] p_{r}(r) d r \\
& =\int_{0}^{R_{e}} f_{0}(r) J(r, \sigma) e^{a^{2} s_{f}^{2} / 2} \frac{2 r}{R_{e}^{2}} d r .
\end{aligned}
$$
by:

In the same way, from Eq. 19, the variance of $f_{u}$ is given

$$
\begin{aligned}
S_{f}^{2} & =\mathbb{E}\left[f_{u}^{2}\right]-\mathbb{E}^{2}\left[f_{u}\right]=\mathbb{E}\left[f_{u}^{2}\right]-M_{f}^{2} \\
\mathbb{E}\left[f_{u}^{2}\right] & =\int \mathbb{E}\left[f_{u}^{2} \mid r\right] p_{r}(r) d r \\
& =\int_{0}^{R_{e}}\left(f_{0}(r) J(r, \sigma)\right)^{2} e^{2 a^{2} s_{f}^{2}} \frac{2 r}{R_{e}^{2}} d r .
\end{aligned}
$$

\section{NOISE AND POWER ANALYSIS}

Recalling Eq. 7, the interference factor term has been fully characterized in the previous section. We now turn to the thermal noise contribution, $h_{u}$.

\section{A. Without shadowing}

In this simple case, $h_{u}=h_{0}=\frac{N_{t h}}{P_{\max } K r_{u}^{-\eta}}$ is a deterministic function at a given distance $r_{u}$. We thus simply need to average this value over the cell area in order to take into account mobile random locations.

$$
\begin{aligned}
\mu_{h_{0}} & =\frac{N_{t h}}{P_{\max } K} \mathbb{E}\left[r_{u}^{\eta}\right] \\
& =\frac{2 N_{t h} R_{e}^{\eta}}{P_{\max } K(\eta+2)} .
\end{aligned}
$$

In the same way:

$$
\begin{aligned}
\sigma_{h_{0}} & =\left(\frac{N_{t h}}{P_{\max } K}\right)^{2} \mathbb{E}\left[r_{u}^{2 \eta}\right] \\
& =\frac{R_{e}^{2 \eta}}{\eta+1}\left(\frac{N_{t h}}{P_{\max } K}\right)^{2}
\end{aligned}
$$

According to Eq. 10 and as $f_{0}$ and $h_{0}$ are not independent, we need to compute:

$$
\begin{aligned}
\mathbb{E}\left[f_{0} h_{0}\right] & =\frac{N_{t h}}{P_{\max } K} \mathbb{E}\left[f_{0}\left(r_{u}\right) r_{u}^{\eta}\right] \\
& =\frac{2 N_{t h} \pi \rho_{B S}}{P_{\max } K(\eta-2)} \mathbb{E}\left[r_{u}^{2 \eta}\left(2 R_{c}-r_{u}\right)^{2-\eta}\right]
\end{aligned}
$$

with

$$
\mathbb{E}\left[f_{0}\left(r_{u}\right) r_{u}^{\eta}\right]=\int_{0}^{R_{e}} r^{2 \eta}\left(2 R_{c}-r\right)^{2-\eta} p_{r}(r) d r .
$$

This integral can be expressed with a formula involving the hypergeometric function but it is not given here for the sake of simplicity.

\section{B. Shadowing impact}

The thermal noise factor is now $h_{u}=h_{0} / A_{b}=h_{0} 10^{-\xi_{b} / 10}$. At a distance $r_{u}$ from the serving BS, this is thus a lognormal RV. Assuming uniform random mobile locations, we have:

$$
\begin{aligned}
M_{h} & =\mu_{h_{0}} \mathbb{E}\left[10^{-\xi_{b} / 10}\right] \\
& =\mu_{h_{0}} e^{a^{2} \sigma^{2} / 2}
\end{aligned}
$$

and

$$
\begin{aligned}
S_{h}^{2} & =\mathbb{E}\left[h_{0}^{2}\right] \mathbb{E}\left[10^{-2 \xi_{b} / 10}\right]-M_{h}^{2} \\
& =\left(\sigma_{h_{0}}^{2}+\mu_{h_{0}}^{2}\right) e^{2 a^{2} \sigma^{2}}-M_{h}^{2} .
\end{aligned}
$$

According to Eq. 13 and keeping in mind that $f_{u}$ and $h_{u}$ are not independent $\mathrm{RV}$, we still need to compute $\mathbb{E}\left[f_{u} h_{u}\right]$ :

$$
f_{u} h_{u}=\frac{N_{t h}}{P_{\max } K} \frac{\sum_{j \neq b}^{B} A_{j} r_{j, u}^{-\eta}}{A_{b}^{2} r_{b, u}^{-2 \eta}} .
$$

With similar arguments as in section IV-B, we see that $f_{u} h_{u}$ can be approximated, at a given distance $r_{u}$, by a lognormal RV. Using the Fenton-Wilkinson method as in [14], we show that the average value of $f_{u} h_{u}$ at distance $r_{u}$ is given by:

$$
\mathbb{E}\left[f_{u} h_{u} \mid r_{u}\right]=\frac{N_{t h}}{P_{\max } K} f_{0} r_{u}^{\eta} e^{3 a^{2} \sigma^{2} / 2} .
$$

We are now able to integrate this equation over the cell area:

$$
\begin{aligned}
\mathbb{E}\left[f_{u} h_{u}\right]= & \int_{0}^{R_{e}} \mathbb{E}\left[f_{u} h_{u} \mid r\right] p_{r}(r) d r \\
= & \frac{4 \pi \rho_{B S} N_{t h} e^{3 a^{2} \sigma^{2} / 2}}{P_{\max } K R_{e}^{2}(\eta-2)} \times \\
& \int_{0}^{R_{e}} r^{2 \eta+1}\left(2 R_{c}-r\right)^{2-\eta} d r .
\end{aligned}
$$

Again, the last integral can be explicitly expressed with the hypergeometric function but the expression is not detailed here for a reason of room. 


\section{ApPlications}

We present in this section applications of our analysis. We first show the influence of the BS output power on capacity on one hand, on coverage on the other hand. Then, for a typical output power, we give the inter-BS distance from which thermal noise is not negligible anymore. At last, we show that BS densification reduces the global power density in the network.

We consider urban and rural environments (see Tab. [15]) and two frequency bands $(920 \mathrm{MHz}$ and $2 \mathrm{GHz})$. We assume voice service in a CDMA network $\left(\gamma^{*}=-19 \mathrm{~dB}\right)$ and the following parameters: $W=5 \mathrm{MHz}, \alpha=0.6, \varphi=0.2, N_{0}=$ $-174 \mathrm{dBm} / \mathrm{Hz}, t=0.5, \eta=3.41$.

Table I

PROPAGATION PARAMETERS

\begin{tabular}{|c|c|c|c|c|c|}
\hline & $\mathrm{K}(2 \mathrm{GHz})$ & $\mathrm{K}(920 \mathrm{MHz})$ & $\sigma(\mathrm{dB})$ & $t$ & $\eta$ \\
\hline Urban & $4.9510^{-4}$ & $6.2410^{-3}$ & 6 & 0.5 & 3.41 \\
\hline Rural & 0.88 & 4.51 & 4 & 0.5 & 3.41 \\
\hline
\end{tabular}

\section{A. Impact on capacity}

Fig. 1 shows the impact of the BS maximum transmitting power on the system capacity. For a given value of $P_{\max }$, the maximum number of MS per cell, $n_{M S}$, such that the outage probability is less than a target value of $P_{\text {out }}^{*}$ of $5 \%$ is deduced from Eq. 11 MS density is $\rho_{M S}=n_{M S} / \pi R_{e}^{2}$. Inter-BS distance $R_{c}$ is set to $1 \mathrm{Km}$ in urban and $5 \mathrm{Km}$ in rural.

This figure quantifies the loss of capacity observed when transmitting power is decreased. For example, when power goes from $20 \mathrm{dBm}$ to $10 \mathrm{dBm}$, capacity decreases from 5 to $1.5 \mathrm{MS} / \mathrm{Km}^{2}$ (urban, $920 \mathrm{MHz}$ ). It is also shown that output power can be limited without loosing capacity: to $28 \mathrm{dBm}$ (urban, $920 \mathrm{MHz}$ ), $39 \mathrm{dBm}$ (urban, $2 \mathrm{GHz}$ ), $26 \mathrm{dBm}$ (rural, $920 \mathrm{MHz}$ ), or $33 \mathrm{dBm}$ (rural, $2 \mathrm{GHz}$ ), depending on the environment and the frequency band.

Increasing the frequency decreases parameter $K$. For a given inter-BS distance $R_{c}$ (e.g. $1 \mathrm{Km}$ ), the interference factor $f_{u}$ is, in this case, unchanged. Only the noise and power contribution $h_{u}$ is affected. This explains why a higher output power is needed to reach the maximum capacity when frequency increases.

In rural environment, several effects appear compared to the urban case. On one hand, since $R_{c}$ is higher, more power is needed in average per MS (see Eq. 44 and the expression of $h_{u}$ ). On the other hand, a higher $K$ (see e.g. Eq. 24) and a lower $\sigma$ (see e.g. Eq. 27 and 22) implies a higher capacity. With the chosen parameters, the increase of $R_{c}$ has a dominant effect and explains the lower MS density.

\section{B. Impact on coverage}

We now quantify the influence of the BS transmitting power on coverage. We consider a fixed MS density of $\rho_{M S}=5.5 \mathrm{MS} / \mathrm{Km}^{2}$ in urban and $\rho_{M S}=0.4 \mathrm{MS} / \mathrm{Km}^{2}$ in rural and we set the target outage probability to $P_{\text {out }}^{*}=1 \%$.
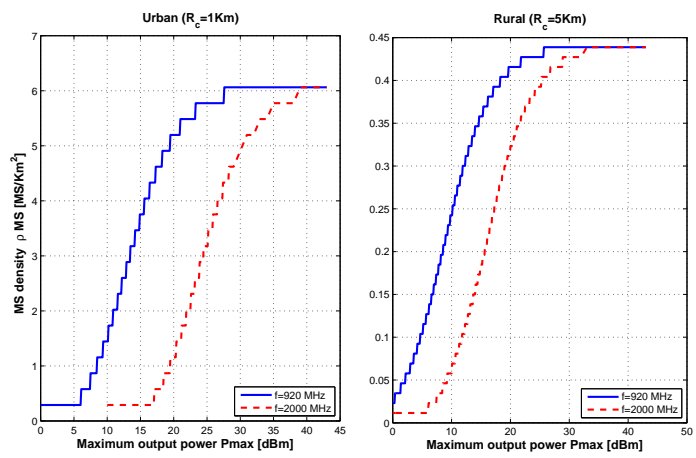

Figure 1. Influence of the output power on capacity.

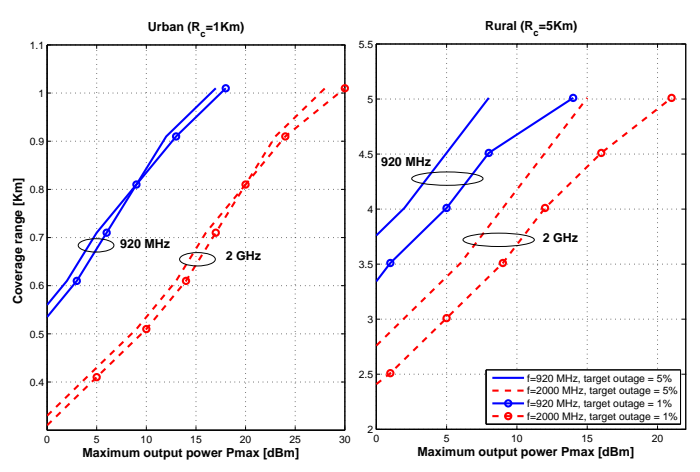

Figure 2. Influence of the output power on coverage.

Half inter-BS distance $R_{c}$ (and so $\rho_{B S}$ ) is constant, but the effective coverage range $R_{e}$ is now variable. Integrations over the cell area in sections IV-B2 and $\mathrm{V}$-B are done on a disk of radius $R_{e}$. Decreasing $R_{e}$ reduces the number of MS per cell and diminishes the average power per user, so that outage probability is decreased.

In our study and for a given output power $P_{\max }$, we search the coverage range for which target outage probability is reached. Results are illustrated in Fig. 2. For example, in urban environment and at $2 \mathrm{GHz}$, when $P_{\max }$ is less than $30 \mathrm{dBm}$, coverage holes appear and at $P_{\max }=20 \mathrm{dBm}$, the coverage range is only $800 \mathrm{~m}$. This figure thus provides the minimum BS power that ensures service continuity.

We now study the effect of a small degradation of the quality of service while setting the target outage to $P_{\text {out }}^{*}=5 \%$. In urban, only 1 and $2 \mathrm{dBm}$ can be saved at 920 and $2 \mathrm{GHz}$. In rural however, important gains can be made. While keeping service continuity (coverage range of $5 \mathrm{Km}$ ), it is possible to reduce the BS power from 14 to $7 \mathrm{dBm}$ at $900 \mathrm{MHz}$ and from 21 to $15 \mathrm{dBm}$ at $2 \mathrm{GHz}$. This gain is obtained at the price of a small degradation of the quality of service.

Of course, these figures depend on the chosen set of parameters and in particular on the propagation model. But they also show that our equations can provide very quick results on the impact of power reduction on the network performance. 

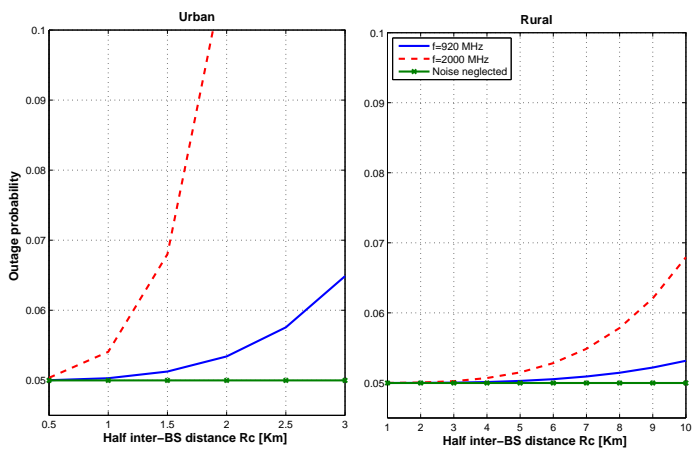

Figure 3. Outage probability vs. half inter-BS distance.

\section{Thermal noise impact on outage}

We now question the traditional assumption of neglecting thermal noise in outage probability computations. We set $P_{\max }=43 \mathrm{dBm}$, which is a standard figure used in cellular networks and we compare in Fig. 3] the outage probabilities obtained with and without considering the thermal noise impact. We set the number of MS per cell, fixed per environment, such that outage probability is $P_{\text {out }}^{*}=5 \%$ when noise is neglected.

When noise is neglected, outage probability depends neither on $K$ nor on the frequency band $\left(h_{u}=0\right.$ in Eq. 6 and $f_{u}$ does not depend on $K$ ). Moreover, it is easy to show with an analysis of Eq. 22 and 23 that varying the inter-BS distance produces homothetic networks, and so, that outage probability does not depend on $R_{c}$.

On the contrary, when noise is not neglected, its contribution is increasing with $R_{c}$. If we accept a error of $0.5 \%$ on the outage, Fig. 3 shows that noise cannot be neglected for $R_{c}>$ $1 \mathrm{Km}$ in urban and $R_{c}>7 \mathrm{Km}$ in rural at $2 \mathrm{GHz}$. Limits are higher at $920 \mathrm{MHz}$.

\section{Power density and BS densification}

We now analyze the global network power density as the function of the BS density in a urban environment. In Fig. 4, MS density is supposed to be constant and set to $\rho_{M S}=5.5 \mathrm{MS} / \mathrm{Km}^{2}$. Full coverage is also assumed. For a given value of $R_{c}$, we compute the minimum maximum power $P_{\max }$ ensuring that outage probability is below $P_{\text {out }}^{*}=5 \%$, power density in the network is obtained with $P_{\max } / \pi R_{e}^{2}$.

Fig. 4 shows that increasing the BS density results in a decrease of the network power density. This effect is particularly important at $2 \mathrm{GHz}$. For example, reducing $R_{c}$ from $1 \mathrm{Km}$ to $950 \mathrm{~m}$ implies a division by almost two of the power density, from $0.46 \mathrm{~W} / \mathrm{Km}^{2}$ to $0.24 \mathrm{~W} / \mathrm{Km}^{2}$. This would mean only a $11 \%$ increase of the number of BS in the network. This also shows that deploying small and femto cells are good means of reducing electromagnetic pollution provided that transmission power is optimized.

\section{CONCLUSION}

In this paper, we derived closed form formulas for outage probabilities in cellular networks, taking into account pathloss, shadowing, thermal noise and random mobile locations.

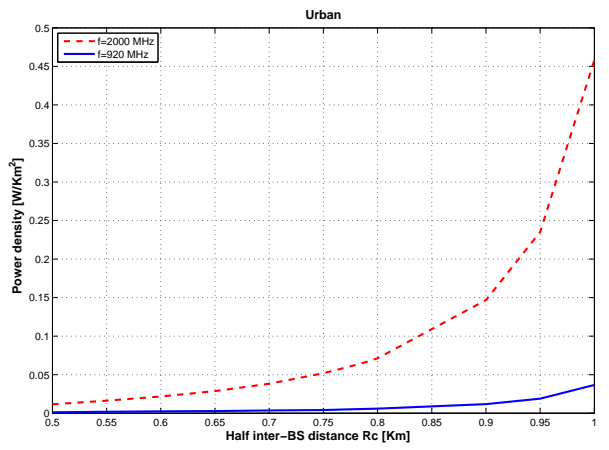

Figure 4. Power density vs. half inter-BS distance.

We showed that these formulas depend on one hand on the interference factor and, on the other hand, on the noise and BS power contribution. This analysis allows the study of the capacity and coverage of a cellular network at a click speed. As an example of it, we studied the influence of the BS power on the network performance. We showed that it is possible to drastically reduce power emissions without loss or with a limited degradation of the quality of service. We also quantified the decrease of the network power density when cell size is reduced.

\section{REFERENCES}

[1] A. J. Viterbi, A. M. Viterbi, and E. Zehavi, Other-Cell Interference in Cellular Power-Controlled CDMA, IEEE Trans. on Communications, Feb/Mar/Apr. 1994.

[2] A. J. Viterbi, CDMA - Principles of Spread Spectrum Communication, Addison-Wesley, 1995.

[3] G. L. Stuber, Principles of Mobile Communications, 2nd Edition, Kluwer Academic Publishers, 2001.

[4] X. Lagrange, Principes et évolutions de l'UMTS, Hermes, 2005.

[5] L. Fenton, The Sum of Lognormal Probability Distributions in Scatter Transmission System, IRE Trans. on Communication Systems, Mar. 1960.

[6] S. Shwartz and Y.S. Yeh, On the Distributions Functions and Moments of Power Sums with Lognormal Components, Bell Syst. Tech. J, Sept. 1982.

[7] Adnan A. Abu-Dayya and Norman C. Beaulieu, Outage Probabilities in the Presence of Correlated Lognormal Interferers, IEEE Trans. on Vehicular Technology, Feb. 1994.

[8] J.-M. Kelif and E. Altman, Downlink Fluid Model of CDMA Networks, Proc. of IEEE VTC Spring, May 2005.

[9] J.-M. Kelif, M. Coupechoux and P. Godlewski, Spatial Outage Probability for Cellular Networks, Proc. of IEEE Globecom Washington, Nov. 2007.

[10] C. C. Chan and Hanly, Calculating the Outage Probabability in CDMA Network with Spatial Poisson Traffic, IEEE Trans. on Vehicular Technology, Jan. 2001.

[11] S. E. Elayoubi and T. Chahed, Admission Control in the Downlink of WCDMA/UMTS, Lect. notes comput. sci., Springer, 2005.

[12] J. S. Evans and D. Everitt, Effective Bandwidth-Based Admission Control for Multiservice CDMA Cellular Networks, IEEE Trans. on Vehicular Technology, Jan. 1999.

[13] J. M. Kelif, Modèle fluide pour les réseaux sans fils, Thèse de doctorat, Télécom ParisTech, 2008.

[14] J.-M. Kelif and M. Coupechoux, Impact of Topology and Shadowing on the Outage Probability of Cellular Networks, Proc. of IEEE ICC, June 2009.

[15] 3GPP, TR 25.816 v7.0.0 - Technical Specification Group Radio Access Network: UMTS $900 \mathrm{MHz}$ Work Item Technical Report (Release 7) (2005-12). 